\title{
NOTE ON ASYMPTOTIC CONTRACTIONS
}

\author{
Ivan D. Aranđelović
}

In $2003 \mathrm{~W}$. A. KIRK introduced the notion of asymptotic contractions. In this paper we present one fixed point theorem of KIRK's type unifying and generalizing recent results of W. A. KIRK, J. JACHYMSKI, I. JóźWIK and Y.-Z. CHEN.

\section{INTRODUCTION AND PRELIMINARIES}

W. A. KIRK [8] introduced the notion of asymptotic contractions and proved fixed point theorem for this class of mappings. In note $[\mathbf{1}]$ we present a new short and simple proof of KIRK's theorem. Further results on this class of mappings was obtained by: J. Jachymski, I. Jóźwik [7], Y.-Z. Chen [4], P. Gerhardy [5], [6], T. Suzuki [9], H. K. Xu [12], M. Arav, F. E. C. Santos, S. Reich, A. Zaslavski [2] and K. Weodarczyk, D. Klim, R. Plebaniak [10], [11]. The papers [10] and [11] presents some ideas for application of the theory of asymptotic contractions in the analysis of set-valued dynamic systems.

In this paper we present one fixed point theorem of KIRK's type unifying and generalizing recent results of W. A. KiRK [8], J. JACHYMsKI, I. JóźwIK [7] and Y.-Z. CHEN [4].

Let $X$ be a nonempty set and $f: X \rightarrow X$ arbitrary mapping. $x \in X$ is a fixed point for $f$ if $x=f(x)$. If $x_{0} \in X$, we say that a sequence $\left(x_{n}\right)$ defined by $x_{n}=f^{n}\left(x_{0}\right)$ is a sequence of PICARD iterates of $f$ at point $x_{0}$ or that $\left(x_{n}\right)$ is the orbit of $f$ at point $x_{0}$.

In [2] M. Arav, F. E. C. Santos, S. Reich and A. Zaslavski proved the following result:

Proposition 1. Let $(X, d)$ be a metric space, $f: X \rightarrow X$ continuous function and $\left(\varphi_{i}\right)$ sequence of functions such that $\varphi_{i}:[0, \infty) \rightarrow[0, \infty)$ and for each $x, y \in X$

$$
d\left(f^{i}(x), f^{i}(y)\right) \leq \varphi_{i}(d(x, y)) .
$$

2000 Mathematics Subject Classification. 54H25, 47H10

Key Words and Phrases. Fixed point, asymptotic contraction. 
Assume also that there exists upper semicontinuous function $\varphi:[0, \infty) \rightarrow[0, \infty)$ such that for any $r>0 \varphi(r)<r, \varphi(0)=0$ and $\varphi_{i} \rightarrow \varphi$ uniformly on any bounded interval $[0, b]$. If there exists $y \in X$ such that $y=f(y)$ then all sequences of Picard iterates defined by $f$ converge to $y$, uniformly on each bounded subset of $X$.

\section{MAIN RESULT'S}

Now we present our results.

Theorem 1. Let $(X, d)$ be a complete metric space, $f: X \rightarrow X$ continuous function and $\left(\varphi_{i}\right)$ sequence of functions such that $\varphi_{i}:[0, \infty) \rightarrow[0, \infty)$ and for each $x, y \in X$

$$
d\left(f^{i}(x), f^{i}(y)\right) \leq \varphi_{i}(d(x, y)) .
$$

Assume also that there exists upper semicontinuous function $\varphi:[0, \infty) \rightarrow[0, \infty)$ such that for any $r>0 \varphi(r)<r, \varphi(0)=0$ and $\varphi_{i} \rightarrow \varphi$ uniformly on any bounded interval $[0, b]$. If one of the following conditions is satisfying:

$$
\begin{aligned}
& \text { 1) there exists } x \in X \text { such that orbit of } f \text { at } x \text { is bounded; or } \\
& \text { 2) } \varliminf_{t \rightarrow \infty}(t-\varphi(t))>0 ; \text { or } \\
& \text { 3) } \varlimsup_{t \rightarrow \infty} \frac{\varphi(t)}{t}<1 .
\end{aligned}
$$

then $f$ has an unique fixed point $y \in X$ and all sequences of Picard iterates defined by $f$ converge to $y$, uniformly on each bounded subset of $X$.

Proof. For any $x, y \in X, x \neq y$, we have:

$$
\varlimsup
$$

Suppose that there exist $x, y \in X$ and $\varepsilon>0$ such that $\varlimsup \lim d\left(f^{n}(x), f^{n}(y)\right)=\varepsilon$. Then there exists sequence of integers $\left(m_{j}\right)$, such that

$$
\lim d\left(x_{m_{j}}, y_{m_{j}}\right)=\varlimsup \lim d\left(x_{m}, y_{m}\right) .
$$

If $\varlimsup \lim d\left(f^{k}(x), f^{k}(y)\right) \geq \varepsilon$, for each $k \in\left(m_{j}\right)$, then from upper semicontinuity of $\varphi$ follows $\varlimsup \lim d\left(x_{m_{j}}, y_{m_{j}}\right) \leq \varphi(\varepsilon)<\varepsilon$, which is a contradiction. So there exists $k \in\left(m_{j}\right)$ such that

$$
\varphi\left(d\left(f^{k}(x), f^{k}(y)\right)\right)<\varepsilon .
$$

This implies that

$$
\begin{aligned}
& \varlimsup \\
& \leq \varlimsup_{n} \varphi_{n}\left(d\left(f^{k}(x), f^{k}(y)\right)\right)=\varphi\left(d\left(f^{k}(x), f^{k}(y)\right)\right)<\varepsilon,
\end{aligned}
$$


which is a contradiction. So we obtain that

$$
\lim d\left(f^{n}(x), f^{n}(y)\right)=0,
$$

for any $x, y \in X$, which implies that all sequences of PICARD iterates defined by $f$, are equiconvergent.

Now let $a \in X$ be arbitrary, $\left(a_{n}\right)$ be a sequence of PICARD iterates of $f$ at point $a, Y=\overline{\left(a_{n}\right)}$ and $F_{n}=\left\{x \in Y: d\left(x, f^{k}(x)\right) \leq 1 / n(k=1, \ldots, n)\right\}$. From (1) follows that $F_{n}$ is nonempty and since $f$ is continuous $F_{n}$ is closed, for any $n$. Also, we have $F_{n+1} \subseteq F_{n}$. Let $\left(x_{n}\right)$ and $\left(y_{n}\right)$ be arbitrary sequences, such that $x_{n}, y_{n} \in$ $F_{n}$. Let $\left(n_{j}\right)$ be a sequence of integers, such that $\lim d\left(x_{n_{j}}, y_{n_{j}}\right)=\varlimsup \lim d\left(x_{n}, y_{n}\right)$.

For any $\varepsilon>0$ there exists positive integer $k$ such that

$$
\varphi(t)+\varepsilon \geq \varphi_{k}(t)
$$

for all $t \in[0,+\infty)$ and $m \geq k$, because $\varphi_{n} \rightarrow \varphi$ uniformly on the rang of $d$. Now we have:

$$
\begin{aligned}
& \lim d\left(x_{n_{j}}, y_{n_{j}}\right) \leq \varlimsup \lim \left(d\left(x_{n_{j}}, f^{n_{j}}\left(x_{n_{j}}\right)\right)+d\left(f^{n_{j}}\left(x_{n_{j}}\right), f^{n_{j}}\left(y_{n_{j}}\right)\right)\right. \\
& \left.+d\left(y_{n_{j}}, f^{n_{j}}\left(y_{n_{j}}\right)\right)\right)=\varlimsup \lim d\left(f^{n_{j}}\left(x_{n_{j}}\right), f^{n_{j}}\left(y_{n_{j}}\right)\right) \\
& \leq \varlimsup \varphi_{n_{j}}\left(d\left(x_{n_{j}}, y_{n_{j}}\right)\right) \leq \varepsilon+\varlimsup \lim \varphi\left(d\left(x_{n_{j}}, y_{n_{j}}\right)\right) \\
& \leq \varepsilon+\varphi\left(\lim d\left(x_{n_{j}}, y_{n_{j}}\right)\right) \text {, }
\end{aligned}
$$

for $n_{j} \geq k$ and so $\lim d\left(x_{n_{j}}, y_{n_{j}}\right)=\varphi\left(\lim d\left(x_{n_{j}}, y_{n_{j}}\right)\right) \Rightarrow \lim d\left(x_{n_{j}}, y_{n_{j}}\right) \in\{0,+\infty\}$. Now we have following three cases:

A) Let $\lim d\left(x_{n_{j}}, y_{n_{j}}\right)=0$. Thus $\varlimsup \lim d\left(x_{n}, y_{n}\right)=0$ and so $\lim d\left(x_{n}, y_{n}\right)=0$. This implies that $\lim \operatorname{diam} F_{n}=0$. By completeness of $Y$ follows that there exists $z \in X$ such that

$$
\bigcap_{i=1}^{\infty} F_{n}=\{z\} .
$$

We remember that 1$) \Rightarrow \mathrm{A})$. Since $d(z, f(z)) \leq 1 / n$ for any $n$, we have $f(z)=z$. From (1) follows that all sequences of PICARD iterates defined by $f$ converge to $z$. From Proposition 1 follows that this convergence is uniform on bounded subsets of $X$.

B) Let $\lim d\left(x_{n_{j}}, y_{n_{j}}\right)=+\infty$ and $\varliminf_{t \rightarrow \infty}(t-\varphi(t))>0$. Then from

$$
d\left(x_{n_{j}}, y_{n_{j}}\right) \leq d\left(x_{n_{j}}, f^{n_{j}}\left(x_{n_{j}}\right)\right)+d\left(f^{n_{j}}\left(x_{n_{j}}\right), f^{n_{j}}\left(y_{n_{j}}\right)\right)+d\left(y_{n_{j}}, f^{n_{j}}\left(y_{n_{j}}\right)\right)
$$

follows

$$
\begin{gathered}
d\left(x_{n_{j}}, f^{n_{j}}\left(x_{n_{j}}\right)\right)+d\left(y_{n_{j}}, f^{n_{j}}\left(y_{n_{j}}\right)\right) \geq d\left(x_{n_{j}}, y_{n_{j}}\right)-d\left(f^{n_{j}}\left(x_{n_{j}}\right), f^{n_{j}}\left(y_{n_{j}}\right)\right) \\
\quad \geq d\left(x_{n_{j}}, y_{n_{j}}\right)-\varphi_{n_{j}}\left(d\left(x_{n_{j}}, y_{n_{j}}\right)\right) \geq d\left(x_{n_{j}}, y_{n_{j}}\right)-\varphi\left(d\left(x_{n_{j}}, y_{n_{j}}\right)\right)-\varepsilon
\end{gathered}
$$


for $n_{j} \geq k$. Thus

$$
\varliminf_{n \rightarrow \infty}(t-\varphi(t))<0
$$

which is a contradiction.

C) Let $\lim d\left(x_{n_{j}}, y_{n_{j}}\right)=+\infty$ and $\varlimsup_{t \rightarrow \infty} \frac{\varphi(t)}{t}<1$. Then from

$$
d\left(x_{n_{j}}, y_{n_{j}}\right) \leq d\left(x_{n_{j}}, f^{n_{j}}\left(x_{n_{j}}\right)\right)+d\left(f^{n_{j}}\left(x_{n_{j}}\right), f^{n_{j}}\left(y_{n_{j}}\right)\right)+d\left(y_{n_{j}}, f^{n_{j}}\left(y_{n_{j}}\right)\right)
$$

follows

$$
\begin{aligned}
1 & \leq \frac{d\left(x_{n_{j}}, f^{n_{j}}\left(x_{n_{j}}\right)\right)+d\left(f^{n_{j}}\left(x_{n_{j}}\right), f^{n_{j}}\left(y_{n_{j}}\right)\right)+d\left(y_{n_{j}}, f^{n_{j}}\left(y_{n_{j}}\right)\right)}{d\left(x_{n_{j}}, y_{n_{j}}\right)} \\
& \leq \frac{d\left(x_{n_{j}}, f^{n_{j}}\left(x_{n_{j}}\right)\right)+\varphi_{n_{j}}\left(d\left(x_{n_{j}}, y_{n_{j}}\right)\right)+d\left(y_{n_{j}}, f^{n_{j}}\left(y_{n_{j}}\right)\right)}{d\left(x_{n_{j}}, y_{n_{j}}\right)} \\
& \leq \frac{d\left(x_{n_{j}}, f^{n_{j}}\left(x_{n_{j}}\right)\right)+\varphi\left(d\left(x_{n_{j}}, y_{n_{j}}\right)\right)+\varepsilon+d\left(y_{n_{j}}, f^{n_{j}}\left(y_{n_{j}}\right)\right)}{d\left(x_{n_{j}}, y_{n_{j}}\right)}
\end{aligned}
$$

for $n_{j} \geq k$. Thus

$$
1 \leq \varlimsup \frac{\varphi\left(d\left(x_{n_{j}}, y_{n_{j}}\right)\right)}{d\left(x_{n_{j}}, y_{n_{j}}\right)}<1
$$

which is a contradiction.

\section{COMMENTS AND REMARKS}

The statement of W. A. KIRK [8] - Theorem 2.1 has additional assumptions that all $\varphi_{i}$ are continuous, and so KIRK's result is include in our Theorem 1.1), as theorem Y.-Z. CHEN [4]- Theorem 2.2 which has additional assumptions that one of $\left(\varphi_{i}\right)$ is upper semicontinuous.

The statement of J. JACHYMSKI, I. Jóźwik [7] - Theorem 2 has additional assumptions " $\mathrm{f}$ is uniformly continuous" and condition

$$
\lim _{t \rightarrow \infty}(t-\varphi(t))=+\infty
$$

which is stronger then our condition

$$
\varliminf_{t \rightarrow \infty}(t-\varphi(t))>0 .
$$

Thus this result is include in our Theorem 1.2.

The statement of Y.-Z. CHEN [4] - Corollary 2.4 has additional assumptions that one of $\left(\varphi_{i}\right)$ is upper semicontinuous, and so it is include in our Theorem 1.3.

In the statements of W. A. KIRK [8] - Theorem 2.1 and Y.-Z. CHEN [4] - Theorem 2.2, the assumption " $f$ is continuous" was inadvertently left out, but 
it was used in the proofs of theorems. J. JACHYMSKI, I. JóźwIK [7], give the following example for necessity of this condition.

Example 1. Let $X=[0,1]$ an $f: X \rightarrow X$ defined by

$$
f(x)= \begin{cases}1, & x=0 \\ x / 2, & x \neq 0\end{cases}
$$

So $f(X) \subseteq(0,1)$ which implies $f^{n}(X) \subseteq\left(0,1 / 2^{n-1}\right)$. A sequence of functions $\varphi_{n}=1 / 2^{n-1}$ satisfies the conditions of Theorem 2.1 because $\varphi_{n} \rightarrow 0$ uniformly, but $f$ is fixed point free.

Now we give the following example for necessity of uniformly convergence of sequence $\left(\varphi_{n}\right)$.

EXAMPLE 2. Let $\left(x_{n}\right)$ be an arbitrary sequence, $X=\left\{x_{n}\right\}$, and $d: X^{2} \rightarrow[0,+\infty)$ mapping defined by

$$
d\left(x_{n}, x_{n+k}\right)=\frac{k}{n+k}+\frac{1}{n k},
$$

$n \in\{1,2,3, \ldots\}, k \in\{0,1,2, \ldots\} .(X, d)$ is complete metric space, because each ball which radius is less then 1 contains only a finite number elements of $X . d$ is discrete metric and all nonzero distance are distinct. Also we have:

$$
\lim _{k} d\left(x_{n}, x_{n+k}\right)=1
$$

and

$$
\lim _{n} d\left(x_{n}, x_{n+k}\right)=0 .
$$

Now define $f: X \rightarrow X$ by $f\left(x_{n}\right)=x_{n+1}$. Let $\varphi(t)=t / 2$ and

$$
\varphi_{n}\left(d\left(x_{i}, x_{j}\right)\right)=\max \left\{d\left(x_{i+n}, x_{j+n}\right), \frac{d\left(x_{i}, x_{j}\right)}{2}\right\},
$$

for $i \neq j$. The function $\varphi_{n}$ is well defined because the number $d\left(x_{i}, x_{j}\right)$ occurs only once in the rang of $d$. From

$$
\lim _{n} d\left(x_{i+n}, x_{j+n}\right)=0
$$

follows $\varphi_{n} \rightarrow \varphi$ on the rang of $d$. The inequality

$$
d\left(f^{n}\left(x_{i}\right), f^{n}\left(x_{j}\right)\right) \leq \varphi_{n}\left(d\left(x_{i}, x_{j}\right)\right)
$$

is also satisfied, but $f$ is fixed point free. 


\section{REFERENCES}

1. I. AranĐelović: On a fixed point theorem of Kirk. J. Math. Anal. Appl., 301 (2005), 384-385.

2. M. Arav, F. E. C. Santos, S. Reich, A. Zaslavski: A note on asymptotic contractions. Fixed point theory and applications (2007) ID 39465, 6 pages, doi: $10.1155 / 2007 / 39465$

3. E. M. BRISEID: A rate of convergence for asymptotic contractions. J. Math. Anal. Appl., (2006) doi:10.1016/jmaa.2006.07.069

4. Y.-Z. Chen:Asymptotic fixed points for nonlinear contractions. Fixed point Theory Appl., 2005:2 (2005), 213-217.

5. P. Gerhardy:A quantitative version of Kirk's fixed point theorem for asymptotic contractions. J. Math. Anal. Appl., 316 (2006), 339-345.

6. P. Gerhardy:Applications of proof interpretations. PhD Dissertation, University of Aarhus 2006.

7. J. JaChymski, I. JóźwiK: On Kirk's asymptotic contractions. J. Mat. Anal. Appl., 300 (2004), 147-159.

8. W. A. KiRK:Fixed points of asymptotic contractions. J. Math. Anal. Appl., 277 (2003), 645-650.

9. T. SUZUKI :Fixed-point theorem for asymptotic contractions of Meir-Keeler type in complete metric spaces. Nonlinear Analysis, 64 (2006), 971-978.

10. K. Wøodarczyk, D. Klim, R. Plebaniak: Existence and uniqueness of endpoints of closedset-valued asymptotic contractions in metric spaces. J. Math. Anal. Appl., 328 (2007), 46-57.

11. K. Weodarczyk, R. Plebaniak, Cezary Obczyński: Endpoints set-valued dynamical systems of asymptotic contractions of Meir-Keeler type and strict contractions in uniform spaces. Nonlinear Analysis, (2006), doi:10.1016/j.na.2006.07.039

12. H. K. XU:Asymptotic and weakly asymptotic contractions. Indian J. pure appl. Math., 36 (2005), 145-150.

University of Belgrade,

(Received October 27, 2006)

Faculty of Mechanical Engineering,

Kraljice Marije 16,

11000 Beograd,

Serbia

E-mail: iva@alfa.mas.bg.ac.yu 